\title{
Abandono do tratamento e cessação do tabagismo: análise dos dados de um programa de controle*
}

Abandonment of treatment and smoking cessation: analysis of data from a control program

Abandono del tratamiento de la cesación tabáquica: análisis de datos de un programa de control

\section{Bruna Vanti da Rocha ${ }^{\mathrm{I}}$, Danielle Soares Rocha Vieira ${ }^{\mathrm{II}}$, Ione Jayce Ceola Schneider ${ }^{\mathrm{III}}$}

\begin{abstract}
Resumo: Objetivo: analisar o perfil dos tabagistas em tratamento e os fatores associados ao abandono do tratamento e cessação do tabagismo em um programa municipal. Método: estudo transversal realizado em Araranguá, Santa Catarina com 109 pessoas do Programa Controle do Tabagismo, entre março e dezembro de 2018. Foram coletadas informações sociodemográficas e de saúde, incluindo sintomas depressivos, impulsividade e capacidade de enfrentamento. Realizouse análises descritivas e inferenciais. Resultados: $40,4 \%$ dos participantes abandonaram o tratamento, grupo que obteve maior média para impulsividade. Os principais motivos relatados para o abandono do tratamento foram: "estar bem sem fumar", situações relacionadas ao trabalho e sintomas de abstinência. Observou-se que 7,64\% cessaram o tabagismo e 93,8\% apresentavam dependência leve à nicotina. Conclusão: apesar do abandono ser alto, alguns permaneceram em cessação. Os com 49 anos ou menos tiveram abandono significativamente maior e a dependência à nicotina apresenta-se como dificultador significativo da cessação.
\end{abstract}

Descritores: Tabagismo; Abandono do Uso de Tabaco; Reabilitação; Saúde Pública; Saúde Mental

Abstract: Objective: to analyze the profile of smokers undergoing treatment and the factors associated with smoking cessation and smoking cessation in a municipal programo of treatment. Method: cross-sectional study carried out in Araranguá, state of Santa Catarina, with 109 people from the Tobacco Control Program, between March and December of the year 2018. Sociodemographic and health information, including depressive symptoms, impulsivity and coping skills, were collected. Descriptive and inferential analyzes were carried out. Results: $40.4 \%$ of the participants abandoned the treatment, group that obtained the highest mean for impulsivity. The main reasons reported for abandoning treatment were: "being well without smoking", work-related situations and withdrawal symptoms. It was observed that $7.64 \%$ quit smoking and $93.8 \%$ had mild dependence on nicotine.

\footnotetext{
I Enfermeira, Mestre, Universidade Federal de Santa Catarina, Araranguá, SC, Brasil. E-mail: brunavantirocha@gmail.com, Orcid: https://orcid.org/0000-0003-2787-6136

II Fisioterapeuta, Doutora, Universidade Federal de Santa Catarina, Araranguá, SC, Brasil. E-mail: danielle.vieira@ufsc.br, Orcid: https://orcid.org/0000-0001-6222-6142

III Fisioterapeuta, Pós-doutora, Universidade Federal de Santa Catarina, Araranguá, SC, Brasil. E-mail: ione.schneider@ufsc.br, Orcid: https://orcid.org/0000-0001-6339-7832.
}

* Extraído da Dissertação “Análise do abandono do tratamento antitabagismo em Araranguá - SC”, Programa de Pós-Graduação em Ciências da Reabilitação, Universidade Federal de Santa Catarina, 2020. 
Conclusion: although abandonment is high, some remained in cessation. Those aged 49 years or less were significantly more abandoned and nicotine addiction is a significant impediment to cessation.

Descriptors: Smoking; Tobacco Use Cessation; Rehabilitation; Public health; Mental Health

Resumen: Objetivo: analizar el perfil de los tabaquistas en tratamiento y los factores asociados al abandono del tratamiento y cesación del tabaquismo en un programa municipal. Método: estudio transversal realizado en Araranguá, Santa Catarina con 109 personas Programa Controle del Tabaquismo, entre marzo y diciembre de 2018. Fueron colectadas informaciones sociodemográficas y de salud, incluindo síntomas depresivos, impulsividad y capacidad de enfrentamiento. Se realizó análisis descriptivas e inferenciales. Resultados: 40,4\% de los participantes abandonaron el tratamiento, grupo que obtuvo mayor media para impulsividad. Los principales motivos relatados para el abandono del tratamiento fueron: "estar bien sin fumar", situaciones relacionadas al trabajo y síntomas de abstinencia. Se observo que 7,64\% cesaran el tabaquismo y 93,8\% presentaban dependencia leve a la nicotina. Conclusión: a pesar del abandono ser alto, algunos siguieron con la cesación. Los con 49 años o menos tuvieron abandono significativamente mayor y la dependencia a la nicotina se presentaba como dificultador significativo de la cesación.

Descriptores: Tabaquismo; Abandono del Uso del Tabaco; Rehabilitación; Salud Pública; Salud Mental

\section{Introdução}

O consumo de tabaco é a principal causa evitável de adoecimento e morte no mundo, o que resulta em cerca de oito milhões de mortes por ano. ${ }^{1}$ Segundo o Instituto Nacional do Câncer, no Brasil, até o ano de 2013, pesquisas populacionais demonstraram queda de $34,3 \%$ no número de fumantes adultos. ${ }^{2}$ Dados da Vigilância de Fatores de Risco e Proteção para Doenças Crônicas por Inquérito Telefônico evidenciam que, em 2018, o percentual total de fumantes com 18 anos ou mais no país foi de 9,3\%, 12,1\% em homens e 6,9\% nas mulheres. ${ }^{3}$ No período de 2005 e 2014, mais de 800 mil fumantes tiveram acesso ao tratamento para parar de fumar pelo Sistema Único de Saúde. ${ }^{1}$

A redução do uso do tabaco no Brasil vem trazendo impacto positivo na reabilitação e promoção da saúde da população, o que reflete na queda de $12 \%$ nos períodos de 1990 a 2015 do total de mortes por câncer de pulmão nos homens, bem como na redução de $25 \%$ nas taxas de Doenças Crônicas Não-Transmissíveis no mesmo período. ${ }^{4}$ Dentre as Doenças Crônicas Não-Transmissíveis, as doenças cardiovasculares $(40,5 \%)$ e respiratórias $(29,9 \%)$ obtiveram maiores taxas de redução. ${ }^{5}$

O Ministério da Saúde brasileiro vem desenvolvendo políticas de ação contra o tabaco coordenadas pelo Instituto Nacional do Câncer. Uma das ações é o Programa Nacional de 
3 | Rocha BV, Vieira DSR, Schneider IJC.

Controle do Tabagismo, instituído em 1989, e que apresenta como principais estratégias de ação para controle do tabagismo a promoção de ambientes livres de fumo, divulgação de informações sobre o tabaco, acesso ao tratamento, regulação da propaganda, impostos sobre o tabaco, repressão ao comércio ilícito, entre outros. ${ }^{6}$ As diretrizes terapêuticas preconizadas pela Portaria 761/2016, para a cessação do tabagismo, baseiam-se nas intervenções cognitivas, treinamento de habilidades comportamentais, apoio medicamentoso quando necessário e estímulo a adoção de hábitos saudáveis e prática de atividade física. ${ }^{7}$

Autores afirmam que reconhecer as características dos fumantes que abandonam o tratamento pode auxiliar na implementação de um tratamento personalizado, principalmente na abordagem clínica inicial do fumante..$^{8-10}$ Estudo aponta como causas do abandono do programa de controle do tabagismo questões relacionadas ao local onde o programa é realizado, horário das reuniões e aos profissionais que prestaram assistência, bem como a utilização da terapia cognitivo-comportamental como estratégia única de tratamento. ${ }^{9}$

Os preditores de cessação bem-sucedida do tabagismo incluem fatores sociodemográficos, como idade mais avançada e alta escolaridade. Os fatores de nível individual associados a cessação incluem tentativa prévia de cessação, intenção de abandonar, menor dependência a nicotina, percepção da saúde como boa/excelente e com autoeficácia relativamente alta. ${ }^{10}$

A respeito do abandono do tratamento do tabagismo, tem-se poucas referências na literatura, especialmente sobre as causas que levam o fumante a apresentar tal comportamento. ${ }^{8-10}$ Portanto, faz-se necessário reconhecer estas informações, objetivando melhorar a qualidade do Programa Nacional de Controle do Tabagismo. Assim, os Órgãos de Saúde possuirão subsídios para colaborar com o enfrentamento de um dos maiores desafios da saúde pública mundial, o consumo do tabaco. ${ }^{9}$ Identificar o perfil e as causas do abandono do programa antitabagismo e da cessação são de relevância para os programas de saúde. Relacionar tais motivos com a adesão ao tratamento pode apontar caminhos para diminuir ainda mais as taxas de abandono do 
tratamento, e promover maior qualidade de vida, minimizar riscos cardiorrespiratórios e oncológicos aos usuários.

Nesse sentido, pretende-se responder a seguinte questão de pesquisa: Qual o perfil dos tabagistas e quais são os fatores que se associam ao abandono do tratamento e cessação do tabagismo em um programa municipal? Assim, o presente estudo tem como objetivo analisar o perfil dos tabagistas em tratamento e os fatores associados ao abandono do tratamento e cessação do tabagismo em um programa municipal.

\section{Método}

Trata-se de um estudo transversal realizado no Município de Araranguá, localizado no Extremo Sul do Estado de Santa Catarina (SC). Araranguá, por meio da Secretaria Municipal de Saúde, atua no controle do uso do tabaco com a implantação, em 2008, do Programa de Controle do Tabagismo. É oferecido tratamento com abordagem intensiva para cessação do tabagismo, com 10 sessões de acompanhamento, o que consiste na estruturação de um local específico para o atendimento de tabagistas que desejam parar de fumar. Essa abordagem é realizada na modalidade de grupo de apoio, pois permite o tratamento de um número maior de fumantes.

O município possui uma equipe multidisciplinar, composta por médico psiquiatra, enfermeiro e psicólogo, que atua no Programa de Controle do Tabagismo. A equipe conta com a colaboração de profissionais do Núcleo de Apoio a Saúde da Família (nutricionista e fisioterapeuta) na abordagem de questões relacionadas à alimentação saudável e estímulo à prática de atividade física. Todo tabagista que tem interesse em parar de fumar é inserido no Sistema de Regulação nas Unidades de Saúde e aguarda vaga para integrar o grupo. O tabagista é comunicado por telefone sobre a data de início do grupo de apoio, que é realizado no auditório da Unidade Central Bom Pastor. Os grupos iniciam com média de 40 participantes e tem periodicidade trimestral. 
5 | Rocha BV, Vieira DSR, Schneider IJC.

Foram pesquisados três grupos de tratamento do Programa de Controle do Tabagismo, que realizaram tratamento no período de março a dezembro de 2018, totalizando 109 participantes. Quanto aos critérios de seleção, foram incluídos indivíduos maiores de 18 anos, independente do sexo, que frequentaram ao menos uma sessão do grupo de tratamento em 2018. Foram excluídos os que não compareceram à primeira sessão de tratamento, pois é nesse momento que são fornecidas orientações sobre o formato, a duração e sanadas as principais dúvidas sobre o programa e a pesquisa, assim como, iniciada a coleta de dados da pesquisa.

A coleta de dados deu-se por meio do preenchimento dos instrumentos por entrevista face-aface, adaptado a partir de estudo prévio, ${ }^{9}$ com dados demográficos, morbidades e grau de dependência na primeira sessão de grupo. Na segunda sessão, foram entrevistados sobre o grau de impulsividade e a presença de sintomas depressivos, e este último, reaplicado na quarta sessão.

A impulsividade foi avaliada utilizando-se a escala BIS 11, instrumento composto por 30 itens relacionados à impulsividade e embasados no modelo de Ernst Barratt. Cada participante analisa cada um dos itens, responde, considerando seu próprio comportamento, e classifica de acordo com uma escala do tipo Likert de quatro pontos. O somatório da pontuação das respostas pode variar entre 30 e 120 pontos e valores altos nos escores foram relacionados a comportamentos impulsivos. A escala é validada para adultos brasileiros. ${ }^{11}$

A presença de sintomas depressivos foi verificada pela autoaplicação do Inventário de Depressão de Beck, validado no Brasil e mundialmente aplicado em adultos. Este questionário consiste no preenchimento de 21 itens, incluindo sintomas e atitudes que variam em pontuação de 0 a 3 e o somatório do resultado é classificado em uma das 4 categorizações [não está deprimido (0-9), leve a moderada (10-18), moderada à severa (19-29) e grave (30-63)]. As afirmações abordam a tristeza, pessimismo, sensação de fracasso, falta de satisfação, sensação de culpa, autopunição, autodepreciação, ideias suicidas, crises de choro, irritabilidade, retração 
Abandono do tratamento e cessação do tabagismo: análise dos dados de um pro... 6

social, indecisão, distorção da imagem corporal, inibição para o trabalho, distúrbio do sono, fadiga, perda de apetite, perda de peso, diminuição de libido. ${ }^{12}$

Para estimar o grau de dependência nicotínica, foi aplicado o Questionário de Fagerström, ferramenta de avaliação validada e mundialmente utilizada em fumantes. O questionário é composto por seis perguntas que abordam o tempo que o fumante leva após acordar para acender o primeiro cigarro, se é difícil ficar sem fumar em ambientes proibidos, qual cigarro do dia traz mais satisfação, quantos cigarros fuma por dia, se fuma mais frequentemente pela manhã e se fuma mesmo doente. Cada resposta possui uma pontuação $(0 \mathrm{a}$ 3) e de acordo com a soma dos pontos obtidos nas perguntas ( 0 a 10) classificou-se a dependência nicotínica em três graus: leve, moderada e severa. ${ }^{13}$

No último encontro do tratamento, com o objetivo de avaliar o enfrentamento a situações estressantes, foi aplicada a Escala Toulousiana de Coping (ETC-R), versão reduzida. A escala é autoaplicável e composta por cinco fatores: (1) controle: evidencia a reação ao estresse, ou seja, quando a pessoa diante de uma situação tem necessidade de refletir e projetar quais as melhores estratégias para utilizar na resolução com eficácia da situação, sem evitar o confronto (itens: 1 , $6,9,15,18)$; (2) recusa: a pessoa procura encontrar uma certa distância cognitiva e emocional face a resolução da situação/problema, podendo até agir recusando-se a admitir a realidade (itens: 10, 11, 12, 16); (3) conversão: estratégia empregada por quem utiliza-se da mudança de comportamento e adaptação da sua ação a situação/problema, responsabilizando-se pela gerência de seus problemas (itens: 4, 5, 14); (4) suporte social: estas pessoas necessitam de conselhos, informações, de ser ouvidas e reconfortadas pelos outros (itens: 7, 8, 13); (5) distração: estratégia em que as pessoas procuram pela companhia de outros ou o envolvimento em atividades que as distraiam dos problemas que as incomodam (itens: $2,3,17$ ). O escore global dá-se pelo somatório dos valores atribuídos aos itens da escala. As manifestações para o estresse de acordo com cada fator dão-se pelo somatório dos escores dos itens descritos. ${ }^{14}$ 
7 | Rocha BV, Vieira DSR, Schneider IJC.

Após o encerramento dos grupos, os abandonos foram identificados e entrevistados em seus domicílios, com o apoio de Agentes Comunitárias de Saúde. O questionário aplicado foi adaptado a partir de estudo anterior e investigou: principal motivo do abandono; local, horário e dia da semana em que o grupo de tratamento é realizado; problemas com a equipe do Programa de Controle do Tabagismo, e tipo de terapia utilizada. Avaliou também se houve indicação médica para o tratamento; se apresenta algum problema de saúde; quantos encontros frequentou; se continua fumando; se mora sozinho ou com mais fumantes na residência. ${ }^{9}$

Considerou-se abandono a ausência em duas ou mais sessões de grupo consecutivas. Durante o acompanhamento dos participantes ocorreu a perda de três indivíduos, o quais não foram localizados por mudança de município.

Os dados foram analisados no programa estatístico Stata 16. Foram realizadas análise descritiva, com medidas de tendência central e dispersão para as variáveis quantitativas, e frequências absolutas e relativas para as variáveis qualitativas, e bivariada com utilização do teste qui-quadrado ou Exato de Fisher para as variáveis qualitativas e Kruskal Wallis, para as quantitativas.

A pesquisa está fundamentada nos princípios éticos da Resolução do Conselho Nacional de Saúde 466 de 12 de dezembro de 2012 e foi aprovada pelo Comitê de Ética e Pesquisa da Universidade Federal de Santa Catarina, sob o Certificado de Apresentação de Apreciação Ética 84197518.0.0000.0121. Todos os participantes consentiram em participar da pesquisa assinando o termo de consentimento livre e esclarecido.

\section{Resultados}

No período em que ocorreu o programa, 163 pessoas estavam inscritas para tratamento, 54 não compareceram na primeira sessão e não foram acompanhados, totalizando 109 participantes que foram incluídos no estudo. O abandono do tratamento foi $40,4 \%$. Nos homens foi de $40,0 \%$ (22) e nas mulheres, de 40,7\% (22). Entre os indivíduos que abandonaram, 6,8\% (3) 
Abandono do tratamento e cessação do tabagismo: análise dos dados de um pro... $\mid 8$

não foram localizados, pois mudaram para outro Município. Em relação as características demográficas, houve prevalência significativamente maior de abandono naqueles com 49 anos ou menos. Já quanto à cessação do tabagismo, 76,4\% pararam de fumar. Não houve diferenças significativas nas condições de saúde e o abandono ao tratamento ou à cessação (Tabela 1).

Tabela 1 - Características, condições e problemas de saúde e suas relações com o abandono do tratamento e cessação do tabagismo, Araranguá, 2018. (n=109)

\begin{tabular}{|c|c|c|c|c|c|}
\hline \multirow[b]{2}{*}{ Variáveis } & \multirow[b]{2}{*}{$\mathrm{n}(\%)$} & \multicolumn{2}{|c|}{ Abandono } & \multicolumn{2}{|c|}{ Cessação } \\
\hline & & $\mathrm{n}(\%)$ & Valor $\mathrm{p}$ & n (\%) & Valor $\mathrm{p}$ \\
\hline \multicolumn{6}{|l|}{ Características } \\
\hline Sexo & & & 0,937 & & 0,736 \\
\hline Masculino & $55(50,5)$ & $22(40,0)$ & & $42(77,8)$ & \\
\hline Feminino & $54(49,5)$ & $22(40,7)$ & & $39(75,0)$ & \\
\hline Faixa etária $(\mathrm{n}=108)$ & & & 0,042 & & 0,150 \\
\hline 49 ou menos & $56(51,9)$ & $28(50,0)$ & & $38(70,4)$ & \\
\hline 50 ou mais & $52(48,1)$ & $16(30,8)$ & & $42(82,4)$ & \\
\hline Estado civil & & & 0,462 & & 0,500 \\
\hline Solteiro & $25(22,9)$ & $12(48,0)$ & & $17(73,9)$ & \\
\hline Separado/Divorciado & $22(20,2)$ & $9(40,9)$ & & $15(71,4)$ & \\
\hline Casado & $51(46,8)$ & $17(33,3)$ & & $42(82,4)$ & \\
\hline Viúvo & $11(10,1)$ & $6(54,5)$ & & $7(63,3)$ & \\
\hline Escolaridade $(\mathrm{n}=107)$ & & & 0,974 & & 0,572 \\
\hline Analfabeto & $6(5,6)$ & $3(50,0)$ & & $4(66,7)$ & \\
\hline $\begin{array}{l}\text { Ensino Fundamental } \\
\text { Incompleto }\end{array}$ & $29(27,1)$ & $12(41,4)$ & & $20(71,4)$ & \\
\hline $\begin{array}{l}\text { Ensino Fundamental } \\
\text { Completo }\end{array}$ & $23(21,5)$ & $10(43,5)$ & & $16(72,7)$ & \\
\hline Ensino Médio Completo & $35(32,7)$ & $13(37,1)$ & & $26(76,5)$ & \\
\hline Ensino Superior Completo & $14(13,1)$ & $6(42,9)$ & & $13(76,0)$ & \\
\hline $\operatorname{IMC}\left(\mathrm{Kg} / \mathrm{m}^{2}\right)(\mathrm{n}=99)$ & & & 0,817 & & 0,853 \\
\hline Normal & $31(31,3)$ & $12(38,7)$ & & $23(79,3)$ & \\
\hline Sobrepeso & $45(45,5)$ & $28(41,2)$ & & $35(79,5)$ & \\
\hline Obesidade & $23(23,2)$ & $40(40,4)$ & & $17(73,9)$ & \\
\hline \multicolumn{6}{|l|}{ Condições de saúde } \\
\hline Atividade física & $23(21,1)$ & $6(26,1)$ & 0,116 & $19(82,6)$ & 0,429 \\
\hline Alterações bucais & $14(12,8)$ & $6(42,9)$ & 0,839 & $12(92,3)$ & 0,150 \\
\hline Diabetes mellitus & $12(11,0)$ & $6(50,0)$ & 0,471 & $9(75,0)$ & 0,902 \\
\hline Hipertensão arterial & $35(32,1)$ & $17(48,6)$ & 0,23 & $24(70,6)$ & 0,332 \\
\hline Doença cardíaca & $13(11,9)$ & $3(23,1)$ & 0,176 & $10(83,3)$ & 0,549 \\
\hline Doença pulmonar & $15(13,8)$ & $7(46,7)$ & 0,592 & $10(71,4)$ & 0,637 \\
\hline
\end{tabular}


9 | Rocha BV, Vieira DSR, Schneider IJC.

\begin{tabular}{lccccc} 
Alteração digestiva & $47(43,1)$ & $18(38,3)$ & 0,701 & $36(81,8)$ & 0,270 \\
Alergia cutânea & $11(10,1)$ & $5(45,5)$ & 0,717 & $8(80,0)$ & 0,779 \\
Câncer & $2(1,8)$ & $1(50,0)$ & 0,779 & $2(100,0)$ & 0,428 \\
Crise convulsiva & $5(4,6)$ & $4(80,0)$ & 0,064 & $3(100,0)$ & 0,329 \\
Anorexia & $5(4,6)$ & $3(60,0)$ & 0,36 & $3(60,0)$ & 0,376 \\
Obesidade & $11(10,1)$ & $4(36,4)$ & 0,775 & $9(81,8)$ & 0,656 \\
Tratamento psicológico & $19(17,4)$ & $11(57,9)$ & 0,087 & $12(66,7)$ & 0,285 \\
Tratamento psiquiátrico & $25(22,9)$ & $12(48,0)$ & 0,376 & $18(75,0)$ & 0,853 \\
Uso de álcool/drogas & $19(17,4)$ & $6(31,6)$ & 0,39 & $14(77,8)$ & 0,881 \\
Outro problema de saúde & $36(33,0)$ & $15(41,7)$ & 0,846 & $26(74,3)$ & 0,717 \\
Uso de medicamento & $71(65,1)$ & $30(42,3)$ & 0,583 & $50(72,5)$ & 0,191 \\
Uso de prótese dentária & $42(38,5)$ & $15(35,7)$ & 0,433 & $31(75,6)$ & 0,877 \\
Uso de ACO* & $6(9,7)$ & $3(50,0)$ & 0,674 & $4(66,7)$ & 0,619 \\
Reposição hormonal & $2(3,2)$ & - & 0,222 & $2(100,0)$ & 0,406 \\
\hline
\end{tabular}

*ACO: anticoncepcional oral

A prevalência de abandonos por grupo de tratamento; a dependência à nicotina; sintomas depressivos antes e após a parada de fumar; os valores da impulsividade e sua subdivisão e enfrentamento a situações estressantes estão apresentados na Tabela 2. Em relação às escalas utilizadas, houve diferença significativa entre cessação e dependência a nicotina, 93,8\% (15) daqueles com dependência leve cessaram o tabagismo enquanto o percentual daqueles com dependência severa foi de $65,1 \%(28)$, representado na Tabela 2.

A impulsividade teve média de 67,4. A área de não planejamento teve maior média entre os fatores. Não foram observadas diferenças nas médias da escala e dos fatores entre os participantes que abandonaram o tratamento ou não, assim como em relação à cessação. O controle foi o item que apresentou maior média em relação ao enfrentamento a situações estressantes para todos os participantes e para os que cessaram o tabagismo. 
Abandono do tratamento e cessação do tabagismo: análise dos dados de um pro... $\mid 10$

Tabela 2 - Características das escalas e suas relações com o abandono do tratamento e cessação do tabagismo, Araranguá, 2018. (n=109)

\begin{tabular}{|c|c|c|c|c|c|}
\hline \multirow[b]{2}{*}{ Variáveis } & \multirow[b]{2}{*}{ n (\%) } & \multicolumn{2}{|c|}{ Abandono } & \multicolumn{2}{|c|}{ Cessação } \\
\hline & & n (\%) & $\begin{array}{l}\text { Valor } \\
\text { de } \mathrm{p}^{*}\end{array}$ & $\mathrm{n}(\%)$ & $\begin{array}{l}\text { Valor } \\
\text { de } \mathrm{p}^{*}\end{array}$ \\
\hline \multicolumn{6}{|l|}{ Características } \\
\hline Grupo (meses de tratamento) & & & 0,774 & & 0,380 \\
\hline Grupo 1 (março a maio) & $33(30,3)$ & $15(45,5)$ & & $21(67,7)$ & \\
\hline Grupo 2 (junho a agosto) & $39(35,8)$ & $15(38,5)$ & & $31(81,6)$ & \\
\hline Grupo 3 (setembro a novembro) & $37(33,9)$ & $14(37,8)$ & & $29(78,4)$ & \\
\hline Dependência à nicotina $(\mathrm{n}=108)$ & & & 0,379 & & 0,048 \\
\hline Leve & $17(15,8)$ & $6(35,3)$ & & $15(93,8)$ & \\
\hline Moderada & $48(44,4)$ & $17(35,4)$ & & $37(80,4)$ & \\
\hline Severa & $43(39,8)$ & $21(48,8)$ & & $28(65,1)$ & \\
\hline Sintomas depressivos antes da parada $(\mathrm{n}=78)$ & & & 0,876 & & 0,714 \\
\hline Não está deprimido & $31(39,7)$ & $10(32,3)$ & & $27(87,1)$ & \\
\hline Depressão leve a moderada & $25(32,1)$ & $8(32,0)$ & & $20(80,0)$ & \\
\hline Depressão moderada a severa & $18(23,1)$ & $4(22,2)$ & & $16(88,9)$ & \\
\hline Depressão severa & $4(5,1)$ & $1(25,0)$ & & $3(100,0)$ & \\
\hline Sintomas depressivos depois da parada $(\mathrm{n}=43)$ & & & 0,771 & & \\
\hline Não está deprimido & $28(65,1)$ & $2(7,1)$ & & - & \\
\hline Depressão leve a moderada & $7(16,3)$ & - & & - & \\
\hline Depressão moderada a severa & $4(9,3)$ & - & & - & \\
\hline \multirow[t]{2}{*}{ Depressão severa } & $4(9,3)$ & - & & - & \\
\hline & $\begin{array}{c}\text { Média } \\
\left(\mathrm{DP}^{\dagger}\right)\end{array}$ & $\begin{array}{c}\text { Média } \\
\left(\mathrm{DP}^{+}\right)\end{array}$ & $\begin{array}{l}\text { Valor } \\
\text { de } p^{*}\end{array}$ & $\begin{array}{c}\text { Média } \\
\left(\mathrm{DP}^{\dagger}\right)\end{array}$ & $\begin{array}{l}\text { Valor } \\
\text { de } \mathrm{p}^{*}\end{array}$ \\
\hline Impulsividade $(\mathrm{n}=68)$ & $\begin{array}{c}67,4 \\
(10,9)\end{array}$ & $69,3(9,2)$ & 0,281 & $65,1(12,6)$ & 0,959 \\
\hline Área motora $(\mathrm{n}=72)$ & $21,4(5,0)$ & $22,0(4,5)$ & 0,453 & $20,2(5,6)$ & 0,519 \\
\hline Área atencional $(\mathrm{n}=70)$ & $18,6(3,5)$ & $19,0(2,8)$ & 0,462 & $18,2(4,1)$ & 0,987 \\
\hline Área não planejamento (n=72) & $27,3(5,1)$ & $28,3(5,0)$ & 0,298 & $26,7(5,6)$ & 0,265 \\
\hline Escala ETC- $R^{*}$ Fator controle $(n=40)$ & $4,0(1,0)$ & - & - & $4,1(1,0)$ & - \\
\hline Escala ETC-R Fator recusa $(\mathrm{n}=38)$ & $3,2(0,9)$ & - & - & $3,3(1,0)$ & - \\
\hline Escala ETC-R* Fator conversão $(n=41)$ & $3,1(0,9)$ & - & - & $3,1(0,9)$ & - \\
\hline Escala ETC- $R^{*}$ Fator suporte social $(n=42)$ & $3,2(1,0)$ & - & - & $3,3(1,1)$ & - \\
\hline Escala ETC- $R^{*}$ Fator distração $(n=41)$ & $3,8(0,9)$ & - & - & $3,9(1,0)$ & - \\
\hline
\end{tabular}

${ }^{\star}$ Escala ETC-R: Escala Toulousiana de Coping

Os principais motivos relatados pelos participantes para abandono do tratamento foram “já estar bem sem fumar" (n=6), situações relacionadas ao trabalho (n=6) e sintomas de abstinência $(\mathrm{n}=5)$. Dos que abandonaram o tratamento, $62,5 \%$ continuavam fumando, $38,2 \%$ 
11 | Rocha BV, Vieira DSR, Schneider IJC.

moram com fumantes e $25 \%$ relataram descontentamento com o local onde o programa era realizado. Os encontros dos 2 primeiros grupos foram realizados na recepção da Unidade Central Bom Pastor, pois o auditório da Unidade encontrava-se interditado. Além disso, 72\% (32) dos que abandonaram o grupo, disseram que participariam novamente.

\section{Discussão}

No ano de 2018, 109 pessoas participaram dos grupos de tratamento antitabagismo promovidos pela Secretaria Municipal de Saúde de Araranguá. O percentual de abandono do tratamento foi de $40,4 \%$ e de cessação, $76,4 \%$. Os motivos relatados pelos participantes para abandono do tratamento referem-se a condições individuais, como não ter a necessidade de continuar o programa para permanecer em abstinência, mas também relacionados à estrutura do local.

O percentual de abandono do tratamento identificado foi relativamente próximo ao relatado no estado de São Paulo, de 30,5\%. ${ }^{15}$ Análise de um Programa de Controle do Tabagismo no Norte do Paraná encontrou percentual de $23,8 \% .{ }^{9}$ No entanto, em Minas Gerais foi observada taxa de $84,6 \%{ }^{16}$ A variação nos valores pode estar relacionada com os critérios utilizados para a definição, como quantidade de faltas, tempo de tratamento e o momento em que foi avaliado. Além disso, o fato de serviços não disponibilizarem gratuitamente a terapia medicamentosa, baixa renda dos participantes, baixo nível educacional e grau elevado de dependência à nicotina, são fatores que podem ter contribuído para essa prática. ${ }^{16}$ Entre os fatores estudados, na presente investigação, os mais jovens tiveram percentual significativamente maior na desistência ao tratamento.

Os fumantes em abandono do tratamento são indivíduos com menos de 49 anos, sexo feminino, economicamente ativos, com elevada dependência a nicotina e não deprimidos, de acordo com Inventário de Beck inicial. Autores relacionam o alto grau de dependência a nicotina como a principal dificuldade encontrada no tratamento antitabagismo, 
Abandono do tratamento e cessação do tabagismo: análise dos dados de um pro... $\mid 12$

consequentemente a maior causa de falha na cessação do tabagismo, pois a dependência é o elemento central na manutenção do tabagismo. ${ }^{17-19}$ A dependência da nicotina possui dois componentes básicos: o físico (desejo intenso de fumar, sintomas de abstinência) e o psicológico (apoio para lidar com situações de solidão, estresse e frustrações). Assim, pessoas com baixa dependência tem menor frequência e intensidade de sintomas de abstinência, logo apresentam maiores chances de sucesso na cessação do tabagismo. ${ }^{18}$

Quanto ao sexo, o dado obtido diverge da literatura, pois outras pesquisas revelaram maior abandono de tratamento pelo sexo masculino. ${ }^{1,9} \mathrm{O}$ dado encontrado nesta pesquisa, com relação as mulheres, pode estar relacionado com as mudanças de humor, o que pode criar dificuldades diferenciadas diante da abstinência do tabaco. Estudo refere também como obstáculo na cessação do tabagismo em mulheres, a preocupação com o peso corporal. ${ }^{20} \mathrm{~A}$ imposição pela sociedade atual de padrões de beleza rígidos quanto as questões estéticas e o uso do tabaco como auxiliar para controle de peso vem sendo reportado na literatura. ${ }^{2}$ Pesquisas evidenciam que são experiências negativas de vida que influenciam as mulheres a fumar. ${ }^{21-22}$ Estudo com mulheres tabagistas discutem o quanto a sobrecarga de trabalho, reprodutivo e produtivo, as incessantes demandas sociais e familiares e as contradições que as mulheres têm vivenciado na sociedade contemporânea podem potencializar o tabagismo feminino. ${ }^{21}$

Dentre as alterações ou condições de saúde relatadas pelos participantes que se obteve maior relação com o abandono do tratamento foi a presença de crises convulsivas e o acompanhamento psicológico prévio. A presença de crises convulsivas contraindica o uso da Bupropiona e levam o fumante a utilizar somente o adesivo de nicotina ou um medicamento de segunda escolha para o controle dos sintomas de abstinência. Isso pode dificultar a parada de fumar, e consequentemente, leva ao abandono do tratamento. ${ }^{7}$

Quanto a impulsividade, as médias foram relativamente maiores entre os que desistiram do tratamento, denotando na área do não planejamento o maior valor. Algumas pessoas, mesmo 
13 | Rocha BV, Vieira DSR, Schneider IJC.

intelectualmente desenvolvidas, podem fracassar em responder adequadamente aos desafios da vida por não conseguirem se organizar. ${ }^{23}$ Uma das hipóteses que vem sendo estudada é a de que o consumo de tabaco seja reflexo de uma busca por “automedicar-se” para alívio dos sintomas decorrentes do Transtorno de Déficit de Atenção, atuando como recurso para melhoria dos processos de atenção e cognição. A prevalência de tabagismo em adolescentes e adultos com Transtorno de Déficit de Atenção tende a ser maior, em comparação a sujeitos sem o transtorno. ${ }^{24}$ No entanto, a pesquisa realizada no Programa de Tabagismo de Araranguá-SC não investigou a presença de casos de Transtorno de Déficit de Atenção nos grupos de tratamento, mas podem estar relacionados a impulsividade.

O principal problema de saúde relatado no momento da entrevista de abandono do tratamento foi a depressão. No entanto, destaca-se que a maioria dos tabagistas que abandonaram o tratamento não foi submetido à segunda aplicação do Inventário de Beck, devido ao abandonado do tratamento antes da aplicação do questionário. Assim, a depressão foi autorreferida pelo entrevistado, sem confirmação diagnóstica. Estudos tem demonstrado que existe forte evidência de relação entre o tabagismo e os transtornos depressivos. ${ }^{25-26}$ Autores encontraram altos níveis de depressão em uma coorte de fumantes na África do Sul. Isso pode demonstrar uma probabilidade de cessação do tabagismo reduzida em fumantes com depressão. Nos tabagistas com histórico de transtornos depressivos o ato de parar de fumar é fator de risco para a manutenção do quadro clínico psiquiátrico, ou mesmo para o desenvolvimento de nova crise depressiva. ${ }^{27}$ Estudo aponta a depressão e/ou ansiedade como segunda comorbidade autorrelatada mais citada e apresentam-se como dificultadores na cessação do tabagismo. ${ }^{28}$

Entre os principais motivos relatados pelos tabagistas no presente estudo para abandono do tratamento estão: situações relacionadas ao trabalho (como alterações no horário, local ou tipo de trabalho), “já estava bem sem fumar” (demonstrando não necessitar mais de medicação e apoio psicológico de grupo) e sintomas de abstinência (dor de cabeça, irritabilidade excessiva, 
Abandono do tratamento e cessação do tabagismo: análise dos dados de um pro... | 14

insônia). O percentual de tabagistas que havia parado de fumar quando foi realizada a entrevista sobre o abandono do tratamento foi superior ao relatado por estudo que identificou que $15,6 \%$ de fumantes desistentes conseguiram interromper o tabagismo sem auxílio. ${ }^{9}$ Assim, o estudo demonstra que mesmo sem concluir o tratamento há sucesso na cessação do tabagismo entre os desistentes.

Entre os motivos relacionados às características da equipe e da estrutura do Programa de Controle do Tabagismo, tem-se o local onde o grupo é realizado com maior percentual. A mudança de local para realização do grupo pode ter contribuído para o abandono do tratamento. Estrutura física inadequada é um dos fatores que contribuem para redução do acesso, da humanização e da continuidade da assistência em saúde. ${ }^{29}$ Assim como, para o profissional de saúde há possibilidade de gerar prejuízos, já que compromete sua autonomia, gerando insatisfação, desgastes, improvisações, conflitos com os usuários, dificuldades para planejar recursos e na realização de práticas com qualidade.

Dentre as limitações do estudo estão as perdas de dados relativos à ausência de alguns participantes no momento da aplicação dos questionários. Como consequência houve redução do tamanho da amostra em algumas medidas realizadas, o que dificultou a comparação. Outra limitação do estudo é que a cessação do tabagismo foi considerada ao final dos grupos de tratamento. Sabe-se que no período de um ano as recaídas são bastante comuns, e considera-se este intervalo para definir a pessoa como ex-fumante.

\section{Conclusão}

Os resultados da pesquisa relativos ao abandono do tratamento oferecem subsídios que justificam a necessidade de modificações na estrutura física do programa, buscando assim oferecer um local adequado e confortável aos participantes e à equipe. Cabe destacar que no grupo dos desistentes havia indivíduos que estavam sem fumar. Isso demonstra que nem todo abandono do tratamento antitabagismo tem relação direta com o insucesso na cessação. 
15 | Rocha BV, Vieira DSR, Schneider IJC.

O fumante em abandono do tratamento para o tabagismo tem o seguinte perfil: menos de 49 anos, viúvo, analfabeto, sexo feminino, economicamente ativo, elevada dependência a nicotina e não está deprimido.

A faixa etária dos fumantes com menos de 49 anos apresentou relação estatisticamente significativa com o abandono do tratamento. O estudo também identifica que questões relacionadas ao acesso aos serviços de saúde impactam na permanência do fumante no tratamento, pois houve abandonos em função de dificuldades de adequar o tratamento à jornada de trabalho de alguns participantes.

Além disso, a dependência à nicotina foi o único dificultador significativo da cessação ao tabagismo. Pessoas identificadas com dependência severa à nicotina devem receber maior atenção dos profissionais da equipe por meio da escuta qualificada e apoio emocional, visto que há maior dificuldade de cessação e permanência no tratamento neste grupo de tabagistas.

\section{Referências}

1. World Health Organization (WHO). Who report on the global tobacco epidemic, 2019: offer help to quit tobacco use [Internet]. Geneva: WHO; 2019 [cited 2020 Jan 20]. Available from: https://apps.who.int/iris/bitstream/handle/10665/325968/WHO-NMH-PND-2019.5-eng.pdf?ua=1

2. Instituto Nacional de Câncer José Alencar Gomes da Silva (INCA). Convenção-quadro da Organização Mundial da Saúde para o controle do tabaco no Brasil: 10 anos de história - 2005-2015 [Internet]. Rio de Janeiro (RJ): INCA; 2018 [acesso em 2020 jan 20]. Disponível em: https://www.inca.gov.br/sites/ufu.sti.inca.local/files//media/document//convencao-quadro-para-ocontrole-do-tabaco-no-brasil-10-anos-de-historia-2005-2015-ed2.pdf

3. Instituto Nacional de Câncer José Alencar Gomes da Silva (INCA). Observatório da Política Nacional de Controle do Tabaco [Internet]. Rio de Janeiro (RJ): INCA; 2019 [acesso em 2020 jan 05]. Disponível em: https://www.inca.gov.br/observatorio-da-politica-nacional-de-controle-do-tabaco/dados-e-numerosprevalencia-tabagismo

4. Guerra MR, Bustamante-Teixeira MT, Corrêa CSL, Abreu DMX, Curado MP, Mooney M, et al. Magnitude e variação da carga da mortalidade por câncer no Brasil e Unidades da Federação, 1990 e 2015. Rev Bras Epidemiol. 2017;20(Suppl 1):102-17. doi: https://doi.org/10.1590/1980-5497201700050009 
Abandono do tratamento e cessação do tabagismo: análise dos dados de um pro... | 16

5. Souza MFM, Malta DC, Franca EB, Barreto ML. Transição da saúde e da doença no Brasil e nas Unidades Federadas durante os 30 anos do Sistema Único de Saúde. Ciênc Saúde Colet [Internet]. 2018 [cited 2020 Jan 20]:1737-50. Available from: https:/www.scielo.br/scielo.php?pid=S1413$81232018000601737 \&$ script $=$ sci_abstract\&tlng=pt

6. Instituto Nacional de Câncer José Alencar Gomes da Silva (INCA). Política Nacional de Controle do Tabaco: relatório de gestão e progresso 2013-2014 [Internet]. Rio de Janeiro (RJ): INCA; 2015 [acesso em 2020 ago 31]. Disponível em: https://www.inca.gov.br/publicacoes/relatorios/politica-nacional-decontrole-do-tabaco-relatorio-de-gestao-e-progresso-2013

7. Ministério da Saúde, Secretaria de Atenção à Saúde. Portaria no 761, de 21 de junho de 2016. Valida as orientações técnicas do tratamento do tabagismo constantes no Protocolo Clínico e Diretrizes Terapêuticas - Dependência à Nicotina. Brasília, DF: Ministério da Saúde, 2016. Disponível em: https://bvsms.saude.gov.br/bvs/saudelegis/sas/2016/prt0761_21_06_2016.html. Acesso em: 21 out. 2019.

8. Bahadir A, Iliaz S, Yurt S, Ortakoylu MG, Bakan ND, Yazar E. Factors affecting dropout in the smoking cessation outpatient clinic. Chron Respir Dis. 2016;13(2):155-61. Epub 2016 Feb 4. doi: https://doi.org/10.1177/1479972316629953

9. Meier DAP, Vannuchi MTO, Secco IAO. Abandono do tratamento do tabagismo em Programa de Município do norte do Paraná. Espaç Saúde [Internet]. 2011 [acesso em 2020 jan 05];13(1):35-44. Disponível em: http://espacoparasaude.fpp.edu.br/index.php/espacosaude/article/view/448

10. Casado L, Thrasher JF, Perez C, Thuler LC, Fong GT. Factors associated with quit attempts and smoking cessation in Brazil: findings from the International Tobacco Control Brazil Survey. Public Health. 2019;174:127-33. doi: https://doi.org/10.1016/j.puhe.2019.06.004

11. Malloy-Diniz LF, Mattos P, Leite WB, Abreu N, Coutinho G, Paula JJ, et al. Tradução e adaptação cultural da Barratt Impulsiveness Scale (BIS-11) para aplicação em adultos brasileiros. J Bras Psiquiatr. 2010;59(2):99-105. doi: https://doi.org/10.1590/S0047-20852010000200004

12. Gorestein C, Andrade LHSG. Inventário de depressão de Beck: propriedades psicométricas da versão em português. Rev Psiquiatr Clín [Internet]. 1998 [acesso em 2018 fev 10];25:245-50. Disponível em: https://www.researchgate.net/publication/284700806_Inventario_de_depressao_de_Beck_Propriedades_p sicometricas_da_versao_em_portugues

13. Halty LS, Hüttner MD, Netto ICO, Santos VA, Martins G. Análise da utilização do questionário de tolerância de Fagerström como instrumento de medida da dependência nicotínica. J Pneumol. 2002;28(4):180-6. doi: https://doi.org/10.1590/S0102-35862002000400002

14. Costa ES, Alves MN, Tap P. Escala Toulousiana de Coping (ETC): estudo de adaptação à população portuguesa. Psicol Saúde Doenças. 2005;6(1):47-56.

15. Longanezi V. Efetividade do programa de tratamento do tabagismo oferecido pelo SUS no Estado de 
São Paulo [dissertação]. São Paulo (SP): Secretaria do Estado de Saúde de São Paulo; 2016 [acesso 2020 jan 19]. Programa de Mestrado Profissional em Saúde Coletiva. Disponível em: http://docs.bvsalud.org/biblioref/2019/07/1006097/valerialonganezi-dissertacaocompleta.pdf

16. Santos SR, Bittencourt ARC, Silva MHC, Stacciarini TSG, Engel RH, Cordeiro ALPC, et al. Perfil e fatores associados ao sucesso terapêutico de tabagistas atendidos em um serviço público especializado. Rev Enferm UERJ. 2018;26:e17381. doi: https://doi.org/10.12957/reuerj.2018.17381

17. Jesus MCP, Silva MH, Cordeiro SM, Korchmar E, Zampier VSB, Merighi MAB. Understanding unsuccessful attempts to quit smoking: a social phenomenology approach. Rev Esc Enferm USP. 2016;50(1):71-8. doi: https://doi.org/10.1590/S0080-623420160000100010

18. Ministério da Saúde (BR). Estratégias para o cuidado da pessoa com doença crônica: o cuidado da pessoa tabagista [Internet]. Brasília (DF): Ministério da Saúde; 2015 [acesso em 2018 dez 12]. (Cadernos da Atenção Básica; 40). Disponível em: http://www.as.saude.ms.gov.br/wpcontent/uploads/2016/06/caderno_40.pdf

19. Chean KY, Goh LG, Liew KW, Tan CC, Choi XL, Tan KC, et al. Barriers to smoking cessation: a qualitative study from the perspective of primary care in Malaysia. BMJ Open. 2019;9(7):1-9. doi: https://doi.org/10.1136/bmjopen-2018-025491

20. Nascimento CCS, Silva GA, Nascimento MI. Fatores associados à recaída do tabagismo em pacientes assistidos em Unidades de Saúde da zona oeste do município do Rio de Janeiro. Rev APS [Internet]. 2016 [acesso em 2019 dez 12];19(4):556-67. Disponível em: https://periodicos.ufjf.br/index.php/aps/article/view/15614

21. Drope J, Schluger NW, Cahn Z, Drope J, Hamill S, Islami F, et al. The tobacco atlas [Internet]. 6th ed. 2018 [cited 2020 Aug 31]. Available from: https://files.tobaccoatlas.org/wpcontent/uploads/2018/03/TobaccoAtlas_6thEdition_LoRes.pdf

22. Reis LM, Gavioli A, Figueiredo VR, Oliveira ML, Efing AC. Uso de tabaco em mulheres acompanhadas em um centro de atenção psicossocial. Acta Paul Enferm. 2019;32(1):27-34.

23. Vasconcelos AG, Teodoro MLM, Malloy-Diniz L, Correa H. Impulsivity components measured by the Brazilian version of the Barratt Impulsiveness Scale (BIS-11). Psicol Reflex Crit. 2015;28(1):96-105. doi: https://doi.org/10.1590/1678-7153.201528111

24. Thakur GA, Sengupta SM, Grizenko N, Choudhry Z, Joober R. Family-based association study of ADHD and genes increasing the risk for smoking behaviours. Arch Dis Child. 2012;97(12):1027-33. doi: https://doi.org/10.1136/archdischild-2012-301882

25. Taylor GMJ, Itani T, Thomas KH, Rai D, Jones $\mathrm{T}$, Windmeijer F, et al. Prescribing prevalence, effectiveness, and mental health safety of smoking cessation medicines in patients with mental disorders. Nicotine Tob Res. 2019;22(1):48-57. doi: https://doi.org/10.1093/ntr/ntz072 
26. Zarghami M, Taghizadeh F, Sharifpour A, Alipour A. Efficacy of smoking cessation on stress, anxiety, and depression in smokers with chronic obstructive pulmonary disease: a randomized controlled clinical trial. Addict Health [Internet]. 2018 [cited 2020 Jan 20];10(3):137-47. Available from: https://www.ncbi.nlm.nih.gov/pmc/articles/PMC6511395/

27. Tadzimirwa GY, Day C, Esmail A, Cooper C, Kamkuemah M, Dheda K, et al. Challenges for dedicated smoking cessation services in developing countries. S Afr Med J. 2019;109(6):431-6. doi: https://doi.org/10.7196/SAMJ.2019.v109i6.13631

28. Pawlina MMC, Rondina RC, Espinosa MM, Botelho C. Depressão, ansiedade, estresse e motivação em fumantes durante o tratamento para a cessação do tabagismo. J Bras Pneumol. 2015;41(5):433-9. doi: https://doi.org/10.1590/S1806-37132015000004527

29. Oliveira MM, Pedraza DF. Contexto de trabalho e satisfação profissional de enfermeiros que atuam na Estratégia Saúde da Família. Saúde Debate. 2019;43(122):765-79. doi: https://doi.org/10.1590/0103-1104201912209

Editora Científica: Tânia Solange Bosi de Souza Magnago

Editora Associada: Maria Denise Schimith

Agradecimento: À Coordenação de Aperfeiçoamento de Pessoal de Nível Superior (CAPES) pelo apoio.

\section{Autor correspondente}

Ione Jayce Ceola Schneider

Endereço: UFSC - Centro de Ciências, Tecnologias e Saúde - Campus Araranguá - Unidade Mato Alto - Rua Pedro João Pereira, 150, Mato Alto - Araranguá - SC CEP 88.905-120

E-mail: ione.schneider@ufsc.br

\section{Contribuições de Autoria}

\section{1 - Bruna Vanti da Rocha}

Concepção ou desenho do estudo/pesquisa, análise e interpretação dos dados, revisão final com participação crítica e intelectual no manuscrito.

\section{2 - Danielle Soares Rocha Vieira}

Análise e interpretação dos dados, revisão final com participação crítica e intelectual no manuscrito.

\section{3 - Ione Jayce Ceola Schneider}

Concepção ou desenho do estudo/pesquisa, análise e interpretação dos dados, revisão final com participação crítica e intelectual no manuscrito.

\section{Como citar este artigo}

Rocha BV, Vieira DSR, Schneider IJC. Abandono do tratamento e cessação do tabagismo: análise dos dados de um programa de controle. Rev. Enferm. UFSM. 2021 [Acesso em: Ano Mês Dia]; vol.11 e41: 1-18. DOI:https://doi.org/10.5902/2179769254535 